\title{
Impacts of motile Escherichia coli on air-water surface tension
}

\author{
Yumeng Zhao ${ }^{1}$, Boyoung Jeong ${ }^{1}$, Dong-Hun Kang ${ }^{1}$, and Sheng Dai ${ }^{1, *}$ \\ ${ }^{1}$ Geosystems Engineering, Georgia Institute of Technology, 790 Atlantic Drive, Atlanta, GA, USA
}

\begin{abstract}
Immiscible multiphase flow in porous media is largely affected by interfacial properties, manifested in contact angle and surface tension. The gas-liquid surface tension can be significantly altered by suspended particles at the interface. Particle-laden interfaces have unique properties, for example, a lower surface tension of interfaces laden with surfactants or nanoparticles. This study investigates the impacts of a motile bacterium Escherichia coli (E. coli, strain ATCC 9637) on the air-water surface tension. Methods of the maximum bubble pressure, the $d u$ Noüy ring, and the pendant droplet are used to measure the surface tension of the motile-bacteria-laden interfaces. Measured surface tension remains independent to the $E$. coli concentration when using the maximum bubble pressure method, decreases with increased $E$. coli concentration in the $d u$ Noüy ring method, and presents time-dependent changes by the pendant drop method. The analyses show that the discrepancies may come from the different convection-diffusion processes of $E$. coli in the flow among various testing methods.
\end{abstract}

\section{Introduction}

Immiscible multiphase flow in porous media happens in many scenarios, such as the drying and wetting of soils, fluid and gas percolation through landfills, hydrocarbon transports through underground rocks, and even in the respiratory and cardiovascular systems in the human body [1-10]. Understanding the immiscible multiphase flow in porous media is of great significance in many engineering applications like hydrocarbon recovery, geological carbon sequestration, nuclear waste disposal, targeted drug delivery, and so on [5-11]. Different instability patterns of multiphase flow in porous media, namely capillary fingering, viscous fingering, and stable displacement, are essentially governed by the contact angle, fluid viscosity, and the flow rate [1].

Preliminary experiments have demonstrated that the presence of a motile bacterium Escherichia coli (E. coli) can significantly alter the drying and wetting processes in a microfluidic chip, yet its underlying physics remains elusive [12]. Many studies have investigated the impacts of active suspensions like motile bacteria to the fluids' rheological behavior [13-14]. However, previous literature has not investigated their impacts to the surface tension or contact angle so that a comprehensive understanding of the effects of motile bacteria to multiphase flow in porous media cannot be achieved. This study presents the first try to probe the effects of motile $E$. coli to the air-water surface tension.

The surface tension may be altered with the presence of suspended particles, no matter actively or passively. The surface tension of interfaces with passive particles of sizes ranging from molecular-scaled surfactants to nanoand micro-particles have been studied [15-17]. However, few investigated the effect of active particles on surface tension. Active particles have different motion inside the bulk fluid and thus different tendencies moving towards or away from the interface. Their adsorption behavior and the ability to change surface tension once adsorbed onto the surface can also be different compared with those of passive counterparts.

We here presents some preliminary studies of the airwater surface tension with the presence of motile $E$. coli (strain ATCC 9637). This bacterium is selected due to its low biosafety risk and well studied motility $[18,19]$. The air-water surface tension is measured in an open-air environment using three different testing methods. The results are also to help understand the underlying mechanisms of surface tension measurements and their ensued impacts on the interpretation of the measured airwater surface tension.

\section{Surface tension measurement methods}

Fig. 1 illustrates six commonly used laboratory methods to measure the water-air surface tension. A detailed discussion on these methods can be found in [20]. Considering the testing accuracy and equipment availability, we use the maximum bubble pressure, the $d u$ Noüy ring, and the pendant drop methods in this study to evaluate the role of motile bacteria on the water-air surface interactions under various testing conditions.

The maximum bubble pressure method is a useful method for measuring the dynamic surface tension [15, 21]. By injecting air at different flow rates through a circular tube into the tested liquid, the bubble can be held

\footnotetext{
* Corresponding author: sheng.dai@ce.gatech.edu
} 
for different durations. During this process, a various amount of suspended particles can adhere to the surface of the bubble as it expands. The surface tension of the fluid can be calculated using the Young - Laplace equation [22] as follows:

$$
\Delta p=p_{1 \max }-p_{2}=\frac{2 \gamma}{r},
$$

where $p_{1 \max }$ is the maximum pressure measured inside the air bubble; $p_{2}$ is the hydrostatic pressure at the depth of the bubble; $\gamma$ is the surface tension; and $r$ is the radius of the injection tubing.

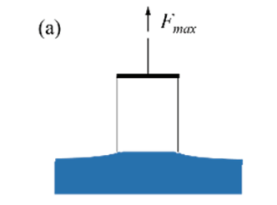

du Noüy ring

(d)

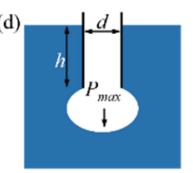

Maximum bubble pressure (b)

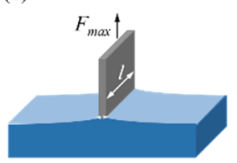

Whilhelmy plate

(e)

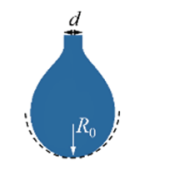

Pendant drop

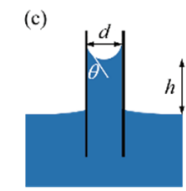

Capillary rise (f)

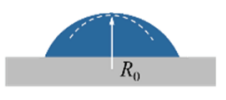

Sessile drop
Fig. 1. Schematic graphs of different surface tension measurement methods (after [23]).

In the $d u$ Noüy ring method, the surface tension is related to the force required to pull a wire ring off the interface $[24,25]$

$$
\gamma=\frac{F_{\max }}{c \cdot \cos \theta}
$$

where $F_{\max }$ is the maximum force applied to lift the ring out of the liquid; $c$ is the perimeter of the three-phase contact line; and $\theta$ is the liquid-air-ring three-phase contact angle.

The pendant drop method captures the profile of a hanging drop. If the best fitted theoretical profile is found, the surface tension value that determines the profile can be back-calculated. The theoretical profile is also determined by the Young - Laplace equation. Detailed expressions for the calculation can be found in [23].

\section{Materials and methods}

The motile bacterium E. coli is grown in a $500 \mathrm{ml}$ nutrient broth medium (ATCC 1376) at $37^{\circ} \mathrm{C}, 160 \mathrm{rpm}$, and $1 \mathrm{~atm}$ conditions for $18 \mathrm{~h}$. Then the microbe suspension is centrifuged (2000 rpm, $10 \mathrm{~min}$ ) and washed twice using the motility buffer solution (MB solution, $0.067 \mathrm{M} \mathrm{NaCl}, 0.1 \mathrm{Mm}$ EDTA, $\mathrm{KH}_{2} \mathrm{PO}_{4}, \mathrm{~K}_{2} \mathrm{HPO}_{4}$ ) to the desired cell concentrations [26]. While not providing any nutrient to the bacteria, the MB solution is used to keep the osmotic and $\mathrm{pH}$ equilibrium as well as the motility of the microbes. Without nutrient, the reproduction and metabolism of the microbes are limited.

Based on our microscopic analysis, the swimming velocity of the studied $E$. coli is on average $4.9 \mu \mathrm{m} / \mathrm{s}$, and does not show any significant change for the duration of the surface tension measurement tests. The volumetric fraction of $E$. coli in the MB solution is determined by counting the colony forming unit (cfu) and assuming each microbe has a volume of $1 \mu \mathrm{m}^{3}$. The prepared microbe suspensions are then tested for their surface tension.

For the maximum bubble pressure method, a glass tube of diameter $0.924 \mathrm{~mm}$ is immersed in the E. coli suspensions and is connected to a syringe. A pressure pump controls the syringe to pump air into the suspension in a constant displacement manner and forms air bubbles. A pressure transducer is added to measure the pressure signature. For the $d u$ Noüy ring method, the Fisher Scientific Model 21 Tensiomat is used. Five repetitive tests on microbe suspension are conducted. And for the pendant drop method, the Ramé Hart tensiometer 250 is used. The drop containing $E$. coli starts with a volume of $8.5 \mu \mathrm{L}$. The surface tension is monitored continuously from the moment the drop is created until the drop is evaporated out.

To demonstrate the surface tension change due to the presence of motile bacteria, MB solutions with a consistent volumetric fraction of $E$. coli between 0.145 $0.162 \%$ are used. All three methods are deployed to measure the surface tension of the MB solution with and without E. coli. Noteworthy that prior to these tests, deionized water is used to calibrate these three methods and all give a consistent value of $72 \mathrm{mN} / \mathrm{m}$. The tests are conducted at a room temperature of $23^{\circ} \mathrm{C}$.

\section{Results}

Fig. 2 shows the surface tension results measured by the maximum bubble pressure method. Here the bubble age means the duration from the moment the bubble is created to the point that the maximum air pressure is obtained. Apparently, a larger pumping rate will result in a shorter bubble age. The maximum bubble pressure result shows that the $0.150 \% \mathrm{E}$. coli suspension has a similar surface tension value with the pure MB solution, which is $72 \mathrm{mN} / \mathrm{m}$. The surface tension is not affected by the bubble age. To illustrate the ability of the maximum bubble pressure method to measure the dynamic surface tension, a solution of $0.2 \%$ mass fraction of commercial detergent is also tested. Because the detergent contains surfactants, a longer bubble age would allow more surfactants to adhere to the air-liquid interface, hence lowering the surface tension. This decreasing trend will end up in a plateau when the adsorption and desorption processes of the surfactants reach an equilibrium. The corresponding surfactant concentration is commonly referred to as the critical micelle concentration (CMC). As expected, the surface tension of the detergent solution decreases with the increasing of the bubble age, and the $\mathrm{CMC}$ is finally reached. Apparently, the E.coli suspension behaves very unlike the detergent solution.

Fig. 3 shows the results obtained by the $d u$ Noüy ring method. Not like the result from the maximum bubble pressure method, the bacteria suspension has a surface tension of $68 \mathrm{mN} / \mathrm{m}$, lower than the pure $\mathrm{MB}$ solution. However, the measured surface tension of MB solution is 
still consistent with the one obtained from the maximum bubble pressure method $(72 \mathrm{mN} / \mathrm{m})$. The surface tension of the detergent solution is $\sim 35 \mathrm{mN} / \mathrm{m}$, in accord with the one measured using the maximum bubble pressure method at a bubble age $\sim 1 \mathrm{~s}$.

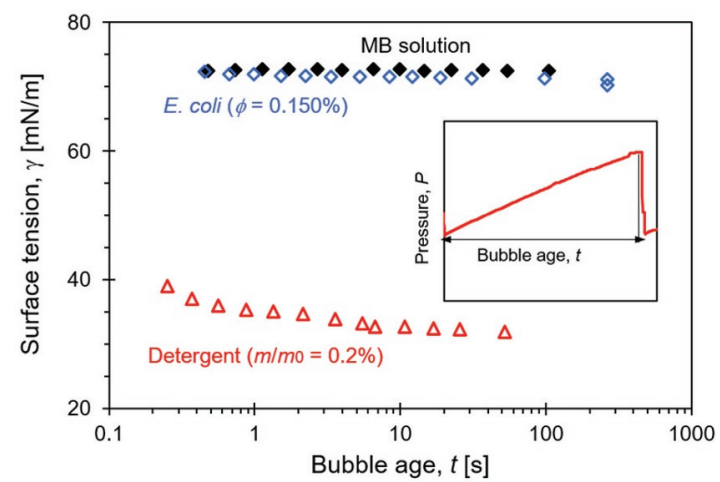

Fig. 2. The surface tension of $E$. coli suspensions measured with the maximum bubble pressure method. Insertion: a typical pressure signature for the life of an air bubble.

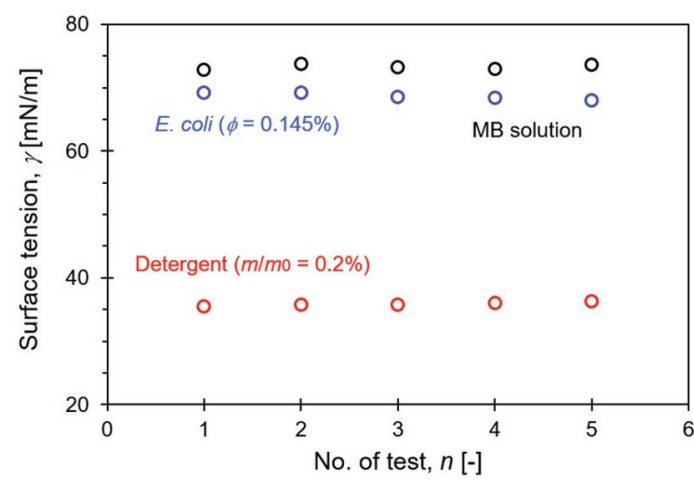

Fig. 3. The surface tension of E. coli suspensions measured with the $d u$ Noüy ring method.

Fig. 4 shows the evolution of the E. coli suspension surface tension over time using the pendant drop method. The volumetric fraction of the microbe is $\phi=0.162 \%$. As the evaporation continues, the volume of the drop keeps decreasing. The surface tension starts from a value of 72 $\mathrm{mN} / \mathrm{m}$, which is equivalent to the $\mathrm{MB}$ solution value, then decreases as evaporating. As the drop shrinks, the profile will be more and more like a perfect circle. This is because that the distortion of the drop shape caused by the selfweight becomes less significant. It is universally agreed that the measured surface tension accuracy would dramatically decrease in this scenario. A dimensionless number, Worthington number $W o$ is a good indicator for the measurement accuracy [23]. Wo is a ratio of the hanging drop weight to the theoretically maximum drop weight that can be achieved and it can be expressed as:

$$
W o=\frac{\Delta \rho g V}{\pi \gamma D}
$$

where $\Delta \rho$ is the density difference inside and outside the droplet; $g$ is the gravitational acceleration; $V$ is droplet volume; and $D$ is the needle diameter. A higher $W o$ number gives a more accurate result. Given this into consideration, we abandon the data when the drop volume shrinks below $2.5 \mu L$. The criterion for the truncation is that below this point, $W o$ is below 0.2. Fig. 4 indicates that when measuring the surface tension of the E.coli suspension by the pendant drop method, the initial value is close to the value of the MB solution $(\phi=0)$; this is in accord with the maximum bubble pressure results. On the other hand, as time goes by, the surface tension decreases; this is in accord with the $d u$ Noüy ring method results. The background group of the MB solution (refer to Fig. 2 and 3) shows no change of surface tension, even the drop is evaporating.

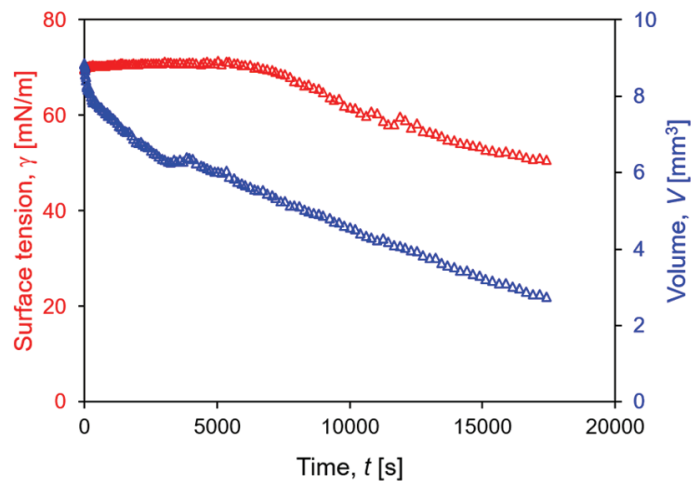

Fig. 4. The surface tension and the drop volume evolution of an $E$. coli suspension measured with the pendant drop method (volumetric fraction $\phi=0.162 \%$ ).

\section{Discussion}

The maximum bubble pressure, the $d u$ Noüy ring and the pendant drop methods are all the commonly used for measuring the surface tension. However, the obtained values may not be consistent with each other. [20] gives an in detailed discussion on the error sources for each of the three methods. For the $d u$ Noüy ring test, the imperfect shape of the ring, the deviation from parallel to the fluid surface when pulling out the ring, and the non-zero contact angle $\theta$ are all possible error sources. High fluid viscosity can also affect the measurement [27]. However, none of the above mentioned are possible reasons for the consistent discrepancy in the experimental data between the MB $(\phi=0)$ solution and $E$. coli suspension $(\phi=$ $0.145 \%$ ). We also do not see any change in the viscosity. For the pendant drop method, anything that can affect the image quality may cause an error, such as poor illumination. A wrong reference scale can also lead to the wrong experimental result. However, in our tests, high image quality and carefully examined scaling are delicately chosen and kept the same for all the tests. Improper needle size or too round a drop will result in inaccurate measurement [23]. Whereas by calculating $W o$, the accurate measurement is also satisfied. For the maximum bubble pressure method, if the injection tubing is not hydrophilic, the escaping bubbles may stick to the tubing surface and affect the measurement [28]. In our 
case, the tubing is hydrophilic glass. If the tubing is not vertical but inclined, the correction for the gravitational deformation of the bubble will be difficult, but the variation is too small that it can be neglected. The possible causes to the discrepancy in the results obtained by these three testing methods are discussed below.

As mentioned, the surface tension data measured by the pendant drop method is similar to the one measured by the maximum bubble pressure method in the beginning and then shift to resemble the $d u$ Noüy ring test result. One reasonable logic is that there are two different dominant physical processes that govern the measured surface tension value. Here we hypothesize that the two governing processes are $E$. coli mass convection along with the flow and the $E$. coli diffusion.

Examine the volume change data by the pendant drop method in Fig. 4, the volume shrinkage rate is almost a constant over the test duration. Thus, we can write as:

$$
V=V_{0}-\alpha t,
$$

where $\alpha$ is a constant. If the pendant drop is always a perfect sphere, then the change of drop radius over time can be derived as:

$$
r=\left(\frac{3}{4 \pi}\left(V_{0}-\alpha t\right)\right)^{\frac{1}{3}} .
$$

In reality, the pendant drop is never a perfect sphere, but the general variation trend for the radius $r$ is similar: the decreasing of $r$ is accelerated over time. If we assume that the change of the evaporation flux at the drop surface with time is negligible, then considering the conservation of mass criterion, a faster shrinkage of drop radius must be compensated by a faster internal flow towards the drop surface. In other words, the convective flow induced by the evaporation becomes stronger and stronger over time. Following this reason, we argue that at the very beginning of the pendant drop measurement, due to the very large droplet radius, the convective flow inside the drop is relatively weak. Therefore, the bacteria cannot be brought to the interface due to the convective flow; the adsorption of the bacteria is mainly due to the diffusion. The passive diffusion of a particle is related to the Brownian motion, which is negligible for a large particle of micrometer scale-like bacteria [29]. In this case, neither the convective flow nor the diffusion is strong enough to bring an extensive amount of the bacteria to the interface. Thus, the surface tension measured by the pendant drop method shows no much change initially. However, with the evaporation goes on, the convective flow becomes stronger and is eventually able to bring the $E$. coli to the interface, hence lowering the surface tension.

For the maximum bubble pressure method, diffusioncontrolled adsorption is the dominant process [21]. When the air pressure pushes to enlarge the bubble, an outward convective flow away from the interface is created. In addition, the diffusion process is suppressed due to the large microbe size. Therefore, limited amount of $E$. coli is expected to attach to the surface, which cannot make a significant change to the measured surface tension value. One prediction is that when the bubble age is long enough, the bubble will be in a quasi-static condition. The convective flow would be negligible, and enough amount of the microbe would have a chance to attach to the interface due to diffusion. At this moment, the change of surface tension will be manifested. This prediction is to be further verified.

Finally, for the $d u$ Noüy ring method, we hypothesize that the pulling of the ring brings a convective flow upwards and brings the E. coli along with the flow. Thus, the attachment of the $E$. coli on the surface reduces the surface tension.

\section{Conclusions}

We measured the surface tension of $E$. coli suspensions with a volumetric fraction of $0.145-0.162 \%$ using three methods: the maximum bubble pressure method, the $d u$ Noüy ring method, and the pendant drop method. The maximum bubble pressure method measures the dynamic surface tension, and measured values show no significant difference in the surface tension between the MB solution with and without $E$. coli. Measured by the $d u$ Noüy ring method, the surface tension of $E$. coli suspension has a mean value of $68 \mathrm{mN} / \mathrm{m}$, lower than the MB solution without $E$. coli. Measured by the pendant drop method, the surface tension of $E$. coli suspension starts from $72 \mathrm{mN} / \mathrm{m}$ which closes to that of the MB solution without $E$. coli. With evaporation, the surface tension decreases over time, possibly due to increased $E$. coli concentration at the air-liquid interface.

The discrepancies in the surface tension measured by the three methods do not reflect the inherent limitations of the techniques as discussed by previous literature. We hypothesis that the non-linear dynamic convectiondiffusion flow in the suspension system contributes to the discrepancies in measured surface tension. This preliminary study on motile $E$. coli laden interface shields light on understanding the active particle laden interface. The study of the active particle laden interface not only extends the physical understanding of multiphase flow in porous media, but also inspires innovative ways of manipulating multiphase flow in porous media, for example, using micro-robots for enhanced nutrient delivery or targeted transport in the fluids.

\section{Acknowledgement}

This material is based upon work primarily supported by the National Science Foundation (EEC-1449501 and CMMI-1943722). Any opinions, findings and conclusions, or recommendations expressed in this material are those of the authors and do not necessarily reflect those of the NSF.

\section{References}

1. R. Lenormand, E. Touboul, C. Zarcone, Numerical models and experiments on immiscible displacements in porous media. J. Fluid Mech. 189, 165-187 (1988) 
2. H. Shin, J.C. Santamarina, Desiccation cracks in saturated fine-grained soils: particle-level phenomena and effective-stress analysis. Geotechnique. 61(11), 961 (2011)

3. D.G. Fredlund, H. Rahardjo. Soil mechanics for unsaturated soils (John Wiley \& Sons, 1993)

4. C.T. Simmons, M.L. Pierini, J.L. Hutson, Laboratory investigation of variable-density flow and solute transport in unsaturated-saturated porous media. Transport Porous Med. 47(2), 215-244 (2002)

5. L.W. Lake, R. Rossen, W.R. Rossen, G.A. Pope. Fundamentals of enhanced oil recovery (2014)

6. L.K. Abidoye, K.J. Khudaida, D.B. Das, Crit. Geological carbon sequestration in the context of two-phase flow in porous media: a review. Rev. Env. Sci. Tec. 45(11), 1105-1147 (2015)

7. R. Jeanloz, H. Stone. Enhanced geothermal systems (EERE Publication and Product Library, 2013)

8. C.F. Tsang, editor. Coupled processes associated with nuclear waste repositories (Elsevier, 2012)

9. D.Y. Yap, D.P. Gaver III, The influence of surfactant on two-phase flow in a flexible-walled channel under bukl equilibrium conditions. Phys. Fluids, 10(8), 1846-1863 (1998)

10. J. Dong, K. Inthavong, J. Tu. Multiphase flows in biomedical applications. In Handbook of multiphase flow science and technology, 1-24 (2017)

11. C.X. Zhao. Multiphase flow microfluidics for the production of single or multiple emulsions for drug delivery. Adv. Drug Deliv. Rev. 65(11-12), 14201446 (2013)

12. B. Jeong, Y. Zhao, D.H. Kang, S. Dai. An experimental study of the effect of motile bacteria on the fluid displacement in porous media. ICEGT Proceedings, La Jolla, CA (2020)

13. K. Son, D.R. Brumley, R. Stocker. Live from under the lens: exploring microbial motility with dynamic imaging and microfluidics. Nat. Rev. Microbiol. 13(12), 761-775 (2015)

14. H.M. López, J. Gachelin, C. Douarche, H. Auradou, E. Clément. Turning bacteria suspensions into superfluids. Phys. Rev. Lett. 115(2), 028301 (2015)

15. J. Eastoe, J.S. Dalton. Dynamic surface tension and adsorption mechanisms of surfactants at the air-water interface. Adv. Colloid Interface Sci. 85(2-3), 103-44 (2000)

16. N. Bizmark, M.A. Ioannidis, D.E. Henneke. Irreversible adsorption-driven assembly of nanoparticles at fluid interfaces revealed by a dynamic surface tension probe. Langmuir, 30(3), 710-717 (2014)

17. K. Du, E. Glogowski, T. Emrick, T.P. Russell, A.D. Dinsmore. Adsorption energy of nano-and microparticles at liquid- liquid interfaces. Langmuir, 26(15), 12518-12522 (2010)
18. J. Schwarz-Linek, J. Arlt, A. Jepson, A. Dawson, T. Vissers, D. Miroli, T. Pilizota, V.A. Martinez, W.C. Poon. Escherichia coli as a model active colloid: A practical introduction. Colloids Surf. B Biointerfaces. 137, 2-16 (2016)

19. N.C. Darnton, L. Turner, S. Rojevsky, H.C. Berg. On torque and tumbling in swimming Escherichia coli. J. Bacteriology, 189(5), 1756-1764 (2007)

20. J. Drelich, C. Fang, C.L. White. Measurement of interfacial tension in fluid-fluid systems. In Encyclopedia of surface and colloid science, 3, 31583163 (2002)

21. N.C. Christov, K.D. Danov, P.A. Kralchevsky, K.P. Ananthapadmanabhan, A. Lips. Maximum bubble pressure method: Universal surface age and transport mechanisms in surfactant solutions. Langmuir, 22(18), 7528-7542 (2006)

22. T. Young. An essay on the cohesion of fluids. Philos. Trans. R. Soc, 95, 65-87, (1805)

23. J.D. Berry, M.J. Neeson, R.R. Dagastine, D.Y. Chan, R.F. Tabor. Measurement of surface and interfacial tension using pendant drop tensiometry. J. Colloid Interface Sci. 454, 226-237 (2015)

24. P. L. du Noüy. A new apparatus for measuring surface tension. J. Gen. Physiol. 1, 521-524 (1919)

25. W.D. Harkins, H.F. Jordan. A method for the determination of surface and interfacial tension from the maximum pull on a ring. J. Am. Chem. Soc. 52(5), 1751-1772 (1930)

26. J. Adler, B. Templeton. The effect of environmental conditions on the motility of Escherichia coli. Microbiol., 46(2), 175-184 (1967)

27. B.B. Lee, E.S. Chan, P. Ravindra, T.A. Khan. Surface tension of viscous biopolymer solutions measured using the du Nouy ring method and the drop weight methods. Polym. Bull. 69(4), 471-489 (2012)

28. K.L. Mysels. Improvements in the maximum-bubblepressure method of measuring surface tension. Langmuir. 2(4), 428-432 (1986)

29. C.J. Van Oss. Interfacial forces in aqueous media (CRC press 2006) 